\title{
High expression of glucose-regulated protein 78 (GRP78) is associated with metastasis and poor prognosis in patients with esophageal squamous cell carcinoma
}

\author{
This article was published in the following Dove Press journal: \\ OncoTargets and Therapy \\ 9 February 2017 \\ Number of times this article has been viewed
}

\section{Peng Ren \\ Chuangui Chen \\ jie Yue \\ Jianguo Zhang \\ Zhentao Yu}

Department of Esophageal Cancer, Tianjin Medical University Cancer Institute and Hospital, National Clinical Research Center of Cancer, Key Laboratory of Cancer Prevention and Therapy of Tianjin City, Tianjin, People's Republic of China
Correspondence: Zhentao Yu Department of Esophageal Cancer, Tianjin Medical University Cancer Institute and Hospital, National Clinical Research Center of Cancer, Key Laboratory of Cancer Prevention and Therapy of Tianjin City, Huanhuxi Road, Tiyuanbei, Hexi District, Tianjin 300060, People's Republic of China

Tel +86 22 23340123

Fax +8622 23359984

Email zhentao_yutj@।63.com
Background: Glucose-regulated protein 78 (GRP78) plays an important role in the invasion and metastasis of many human cancers. However, the role of this protein in the progression of invasion and metastasis in esophageal squamous cell carcinoma (ESCC) remains elusive.

Patients and methods: Immunohistochemistry and Western blot were performed to analyze GRP78 expression in 92 patients with primary ESCC. The correlation of GRP78 expression with clinicopathological factors was analyzed. In vitro, the expression levels of GRP78 were downregulated by small interfering RNA transfection in TE-1 and CaEs-17 ESCC lines. Cell invasion and migration assays were applied to determine the invasion and migratory abilities of ESCC cells.

Results: Compared with GRP78 in adjacent normal esophageal tissues, GRP78 was overexpressed in ESCC tissues. High GRP78 expression was significantly correlated with positive lymph node metastasis $(P=0.035)$ and advanced tumor stage $(P=0.017)$. Survival analysis revealed that high GRP78 expression was significantly associated with shorter overall survival $(P=0.037)$. In multivariate analysis, GRP78 overexpression was identified as an independent prognostic factor for overall survival $(P=0.011)$. si-GRP78 can significantly decrease the GRP78 expression level and reverse the invasion and migratory abilities of ESCC cells in TE-1 and CaEs-17 cell lines.

Conclusion: These findings demonstrated that high expression of GRP78 was associated with disease progression and metastasis in ESCC and might serve as a novel prognostic marker for patients with ESCC.

Keywords: GRP78, esophageal squamous cell carcinoma, metastasis, prognosis

\section{Introduction}

Esophageal cancer is the eighth most common cancer worldwide, and esophageal squamous cell carcinoma (ESCC) accounts for $\sim 90 \%$ of esophageal cancer cases. ${ }^{1}$ Despite advancements in diagnostic and treatment approaches, the clinical outcome for all patients remains poor because of early lymph node (LN) metastasis and invasion of adjacent organs, such as the aorta, trachea, bronchus, pericardium, and lung. The 5-year overall survival (OS) rate of patients diagnosed with esophageal cancer ranges from $15 \%$ to $20 \% .^{2}$ Therefore, in-depth studies on the molecular mechanism of invasion and metastasis in ESCC should be performed and effective prevention and treatment measures should be developed to improve therapeutic outcomes for patients with esophageal cancer. 
Glucose-regulated protein 78 (GRP78), a major constituent of the endoplasmic reticulum, is responsible for protein folding and denaturing to maintain cellular integrity. ${ }^{3}$ GRP78 is expressed at basal levels in normal adult organs but is strongly induced in tumors, ${ }^{4}$ such as malignant gliomas, colon cancer, esophageal cancer, and hepatocellular cancer. ${ }^{5-8}$ Recent advances have revealed that GRP78 overexpression is associated with tumor cell proliferation, invasion, chemotherapeutic response, and patient prognosis in esophageal cancer. ${ }^{9-11}$ Novel therapeutic approaches targeting glucoseregulated proteins have been developed in cancer treatment. ${ }^{11}$ ESCC is a more common type of esophageal cancer in the People's Republic of China than adenocarcinoma in Western countries. ${ }^{2}$ However, most data on GRP78 have been generated from studies on esophageal adenocarcinoma and focused on prognosis or treatment response. ${ }^{9,10}$ Invasion and metastasis have impeded the enhancement of survival rates in the clinical treatment of ESCC. Increased GRP78 expression is also correlated with LN metastasis in gastric cancer $^{12}$ and implicated in the promotion of hepatocellular carcinoma invasion by enhancing focal adhesion kinase (FAK) activation ${ }^{13}$ or by decreasing E-cadherin levels. ${ }^{8} \mathrm{Nev}-$ ertheless, the role of GRP78 in the invasion and metastasis in ESCC has been rarely reported.

Therefore, in this study, GRP78 expression and its clinical implications in ESCC were examined to investigate the effect of GRP78 on invasion, metastasis, and prognosis in patients with ESCC.

\section{Patients and methods}

\section{Patients and treatment}

Ninety-two patients with ESCC who provided complete clinicopathological and follow-up data and underwent surgical resection at Tianjin Medical University Cancer Institute and Hospital between January 2008 and December 2010 were enrolled in this study. None of the patients experienced a previous history of cancer nor received any chemotherapy or radiation before surgery. The protocol of this study was approved by the Research Ethics Committee of Tianjin Medical University Cancer Institute and Hospital, People's Republic of China. Written informed consents were obtained from all the patients.

Tissue samples were fixed in formalin, routinely processed, and embedded in paraffin. All the tumor tissues were confirmed through pathological examination. Histological grading was defined as well, moderately, or poorly differentiated/undifferentiated in accordance with the World Health Organization classification of esophageal tumors. ${ }^{14}$ Tumor stage was determined based on the seventh edition of the American Joint Committee on Cancer
(AJCC) tumor-node-metastasis (TNM) staging system for esophageal carcinoma. ${ }^{15}$ To evaluate the depth of tumor invasion, we considered the following definition: pT1 for tumors that invaded the lamina propria, muscularis mucosae, or submucosa, pT2 for tumors that invaded the muscularis propria, pT3 for tumors that invaded the adventitia, and pT4 for tumors that invaded adjacent structures. LN metastasis was classified on the basis of the number of metastatic LNs as follows: N0, no metastasis; N1, 1-2 metastatic LNs; N2, 3-6 metastatic LNs; and N3, $\geq 7$ metastatic LNs. Patients designated with $\mathrm{pN} 0$ were categorized into the $\mathrm{N} 0$ group and those classified as $\mathrm{pN} 1, \mathrm{pN} 2$, and $\mathrm{pN} 3$ were placed into the $\mathrm{N}+$ group.

The patients were followed up through direct evaluation or phone interviews until death or February 28, 2015. OS was defined as the time from the date of surgery to the date of last contact or the date of death.

\section{Immunohistochemistry}

Consecutive sections ( $6 \mu \mathrm{m}$ thickness) were cut from formalin-fixed and paraffin-embedded tissue blocks. Each slide was deparaffinized with xylene, dehydrated with alcohol, and subjected to heat-mediated antigen retrieval with citrate buffer at $\mathrm{pH} 6$. The sections were then incubated with the primary antibody GRP78 (1:100; Abcam, Cambridge, UK) at $4^{\circ} \mathrm{C}$ overnight. The primary antibodies were subsequently replaced with phosphate buffer saline (PBS) as a negative control. The sections were conjugated with secondary antibodies for $30 \mathrm{~min}$ at room temperature. Diaminobenzidine was used as chromogen, and the sections were subsequently counterstained with hematoxylin, dehydrated, and mounted.

The sections were examined and scored independently by two pathologists who were blinded to the clinicopathological information, and at least five fields were randomly chosen. The GRP78 expression was evaluated on the basis of the ratio of positive cells per specimen and staining intensity. The ratio of positive cells per field was evaluated quantitatively and scored as 0 for staining $<5 \%, 1$ for staining 5\%-25\%, 2 for staining $26 \%-50 \%, 3$ for staining $51 \%-80 \%$, and 4 for staining $>80 \%$. The intensity was graded as follows: 1, weak; 2, moderate; and 3, strong. A final immunohistochemical score was achieved by multiplying the intensity score and the percentage of positive cells (range 0-12). In this study, 92 ESCC patients were divided into high- and low-GRP78-expression groups by determining an optimal cutoff value for GRP78 expression levels on the basis of the measure of heterogeneity by using log-rank test statistic with respect to OS as previously published. The patients were subsequently categorized into the following two groups: high-GRP78-expression group with a staining index score 
of $\geq 7$ and low-GRP78-expression group with a staining index score of $\leq 6$.

\section{Cell line and culture}

The human ESCC lines EC-109, TE-1, and CaEs-17 were purchased from the China Academy of Science cell library and maintained in RPMI-1640 medium supplemented with $10 \%$ fetal calf serum (Gibco, Grand Island, NE, USA) in a humidified atmosphere of $5 \% \mathrm{CO}_{2}$ at $37^{\circ} \mathrm{C}$.

\section{Western blot}

Western blot analysis was performed as previously described to detect the expression levels of GRP78 in esophageal cancer tissues or cell lines. The cells were washed with cold PBS and lysed with RIPA buffer, which contained protease inhibitors. Afterward, $30 \mu \mathrm{g}$ of protein were resolved on a sodium dodecyl sulfate denaturing polyacrylamide gel and transferred onto polyvinylidene fluoride membranes (Millipore, Billerica, MA, USA). The membranes were subsequently blotted using an anti-GRP78 antibody (1:1,000; Abcam) or anti- $\beta$-actin antibodies (1:2,000; Proteintech, Chicago, IL, USA). After these membranes were incubated at $4{ }^{\circ} \mathrm{C}$ overnight, the secondary antibodies (1:2,000; Proteintech) were added, and the resultant mixture was incubated for $1 \mathrm{~h}$ at $4^{\circ} \mathrm{C}$. The immune complexes were visualized with an enhanced chemiluminescence Plus kit (GE, Fairfield, CT, USA) and detected using a VersaDoc 5000 system (Bio-Rad Laboratories, Hercules, CA, USA) in accordance with the manufacturer's protocol.

\section{Small interfering RNA (siRNA) transfection}

The siRNA against GRP78 was prepared as described in a previous study. ${ }^{16}$ A scrambled sequence unrelated to si-GRP78 was used as control. For transfection, TE-1 and CaEs-17 cells were plated in six-well plates containing $2 \mathrm{~mL}$ of the medium supplemented with $10 \%$ fetal bovine serum (FBS). After 18 h, si-GRP78 was transfected by using Lipofectamine ${ }^{\circledR}$ 2000 Reagent (Invitrogen, Carlsbad, CA, USA) when the cells were $50 \%-60 \%$ confluent. The cells were harvested $48 \mathrm{~h}$ after transfection and used for analysis.

\section{Cell migration and invasion assays}

Invasion and migration assays were performed as previously described. ${ }^{17}$ The invasion and migration abilities of TE- 1 and CaEs-17 cells transfected with si-GRP78 and si-con were tested in Matrigel-coated cell culture chambers (Millipore). In brief, $10 \times 10^{5}$ cells were transfected and cultured in invasion chambers with Costar Transwell ${ }^{\circledR} 8 \mu \mathrm{m}$ inserts coated with $50 \mu \mathrm{g}$ of reduced serum Matrigel in accordance with the manufacturer's instructions. The medium supplemented with $10 \%$ FBS was used in the lower chamber. Migration assays were performed in the same manner excluding Matrigel. After $16 \mathrm{~h}$, noninvading cells and media were removed with a cotton swab. The cells on the lower surface of the membrane were fixed with polyoxymethylene and stained with $0.1 \%$ crystal violet for $30 \mathrm{~min}$. The stained cells were counted under a microscope in four randomly selected fields, and the average number was used to indicate cell migration and invasion.

\section{Statistical analysis}

SPSS 17.0 (SPSS Inc., Chicago, IL, USA) was used for statistical analysis. Quantitative variables were compared using the Student's $t$-test and expressed as median \pm standard deviation. The association between GRP78 expression and clinicopathological features of esophageal cancer was analyzed by $\chi^{2}$ test or Fisher's exact test. Survival analysis was performed using the Kaplan-Meier method, and differences in survival were estimated via log-rank test. Significant parameters revealed through univariate analysis were subjected to multivariate survival analysis by using Cox's proportional hazard model. The hazard ratios (HRs) and corresponding 95\% confidence intervals (CIs) were calculated. Two-tailed $P<0.05$ was considered statistically significant.

\section{Results}

\section{Clinicopathological characteristics of ESCC patients}

A total of 67 males and 25 females with a median age of 65 years (range $43-80$ years) were included in this study. Of the 92 cases of ESCC, 46 cases had a lesion length of $<4 \mathrm{~cm}$ and 46 cases had a lesion length of $\geq 4 \mathrm{~cm}$. A total of 55 patients demonstrated no LN metastasis (N0), whereas 37 patients were identified with LN involvement $(\mathrm{N}+)$. For clinical stages, 10 cases were in stage I, 36 cases were in stage II, and 46 cases were in stage III in accordance with the seventh edition of AJCC TNM staging criteria for esophageal cancer.

The follow-up period ranged from 3 to 72 months (median 36 months). By the end of this study, 52 of the 92 patients had died.

\section{GRP78 is overexpressed in ESCC tissues and ESCC cell lines}

GRP78 overexpression has been reported in many kinds of human cancers. However, its expression status in ESCC remains unknown. Western blot analysis was carried out to 


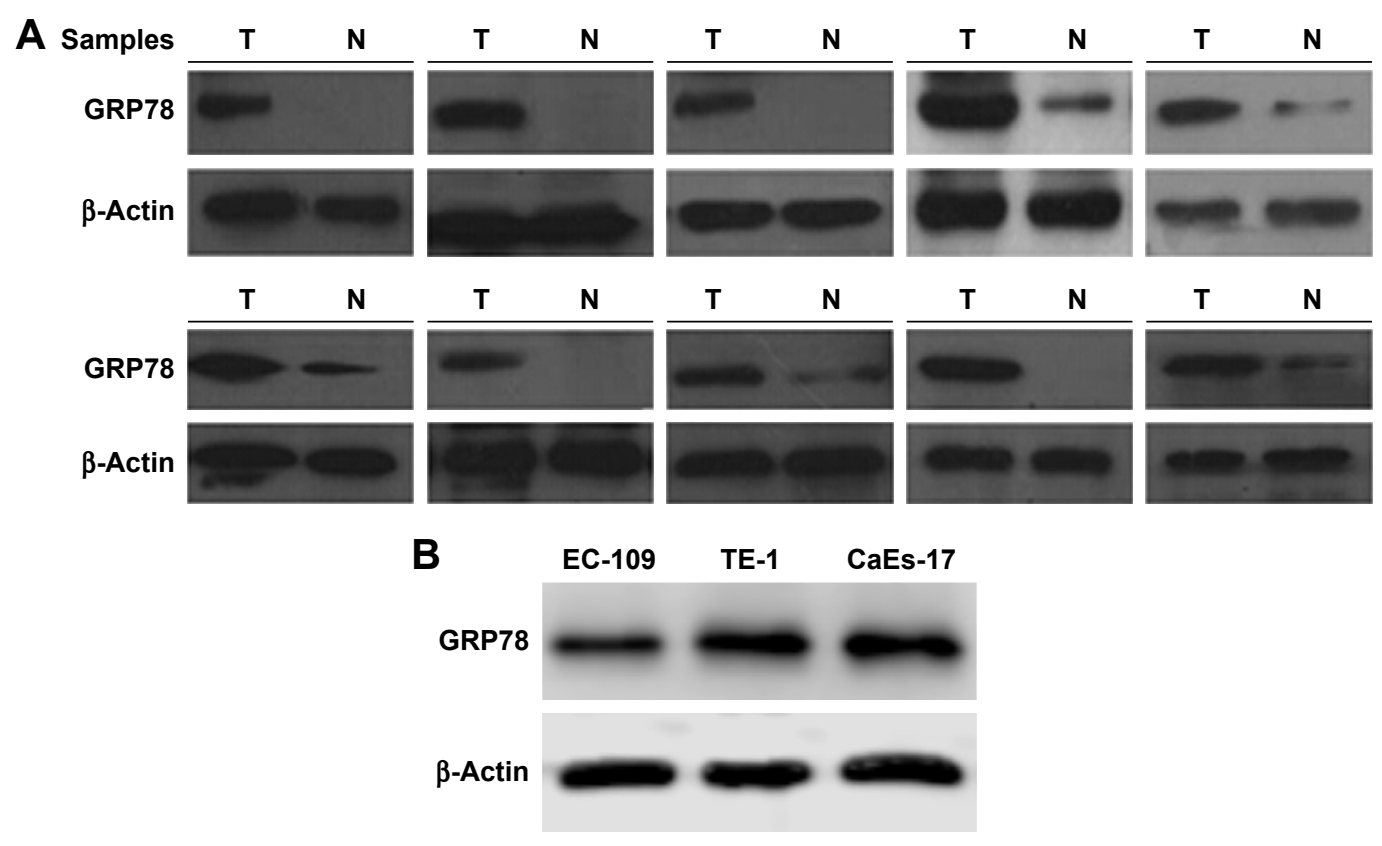

Figure I The expression of GRP78 protein in ESCC tissues and cell lines was assessed through Western blot.

Notes: (A) Western blot results of GRP78 protein levels in ESCC tissues and adjacent normal esophageal mucosal tissues. The GRP78 protein levels are higher in ESCC tissues (T) than in adjacent normal esophageal mucosal tissues (N) from the same patient. (B) Western blot results of GRP78 protein levels in different ESCC cell lines. The GRP78 protein levels are high in the three ESCC cell lines.

Abbreviations: ESCC, esophageal squamous cell carcinoma; GRP78, glucose-regulated protein 78.

evaluate the expression level of GRP78 in ESCC tissues and cell lines. Its expression levels in 12 cases of ESCC specimens were compared with paired adjacent normal esophageal mucosal tissues through Western blot. GRP78 was expressed to a greater extent in ESCC samples than in adjacent normal esophageal tissues (Figure 1A). Furthermore, GRP78 was highly expressed in the three ESCC cell lines, especially in TE-1 and CaEs-17 (Figure 1B).

The expression and localization of GRP78 were evaluated through immunohistochemistry in the samples from 92 patients with ESCC. The expression of GRP78 was primarily localized in the cytoplasm of the carcinoma cells in ESCC tissues. A total of 66 of the 92 cancer lesions (71.7\%) were scored as high expression, but 20 of the 92 cases (21.7\%) were scored as high expression in adjacent normal esophageal mucosal tissues, and their difference was significant $(P<0.05)$. The representative results of GRP78 staining are shown in Figure 2.

\section{Correlation of GRP78 expression with the clinicopathological factors in patients with ESCC}

We then explored the association of GRP78 expression with clinicopathological parameters in the 92 cases of ESCC patients. In Table 1, high GRP78 expression was closely correlated with positive LN metastasis $(P=0.035)$ and advanced tumor stage $(P=0.017)$. Conversely, the GRP78 expression was not correlated with gender $(P=0.627)$, age ( $P=0.165)$, smoking status $(P=0.745)$, alcohol consumption $(P=0.466)$, tumor location $(P=0.453)$, histological grade $(P=0.191)$, tumor size $(P=0.165)$, and tumor invasion $(P=0.213$; Figure 3$)$.

\section{GRP78 expression and prognosis in patients with ESCC}

The cancer patients were subjected to follow-up examinations for up to 72 months, with a median follow-up period of 36 months (range 3-72 months). The 5-year survival rate was $30.7 \%$ for all of the patients. The 5 -year survival rate and the median survival time of the patients with low GRP78 expression were $42.3 \%$ and 48 months, respectively. The 5-year survival rate and the median survival time of the patients with high GRP78 expression were $26.0 \%$ and 28 months, respectively. Their difference was significant (log-rank test: $P=0.037$ ).

Table 2 lists the results of the univariate and multivariate analyses of the factors related to the prognosis of patients with ESCC. Univariate analysis with Cox's proportional 

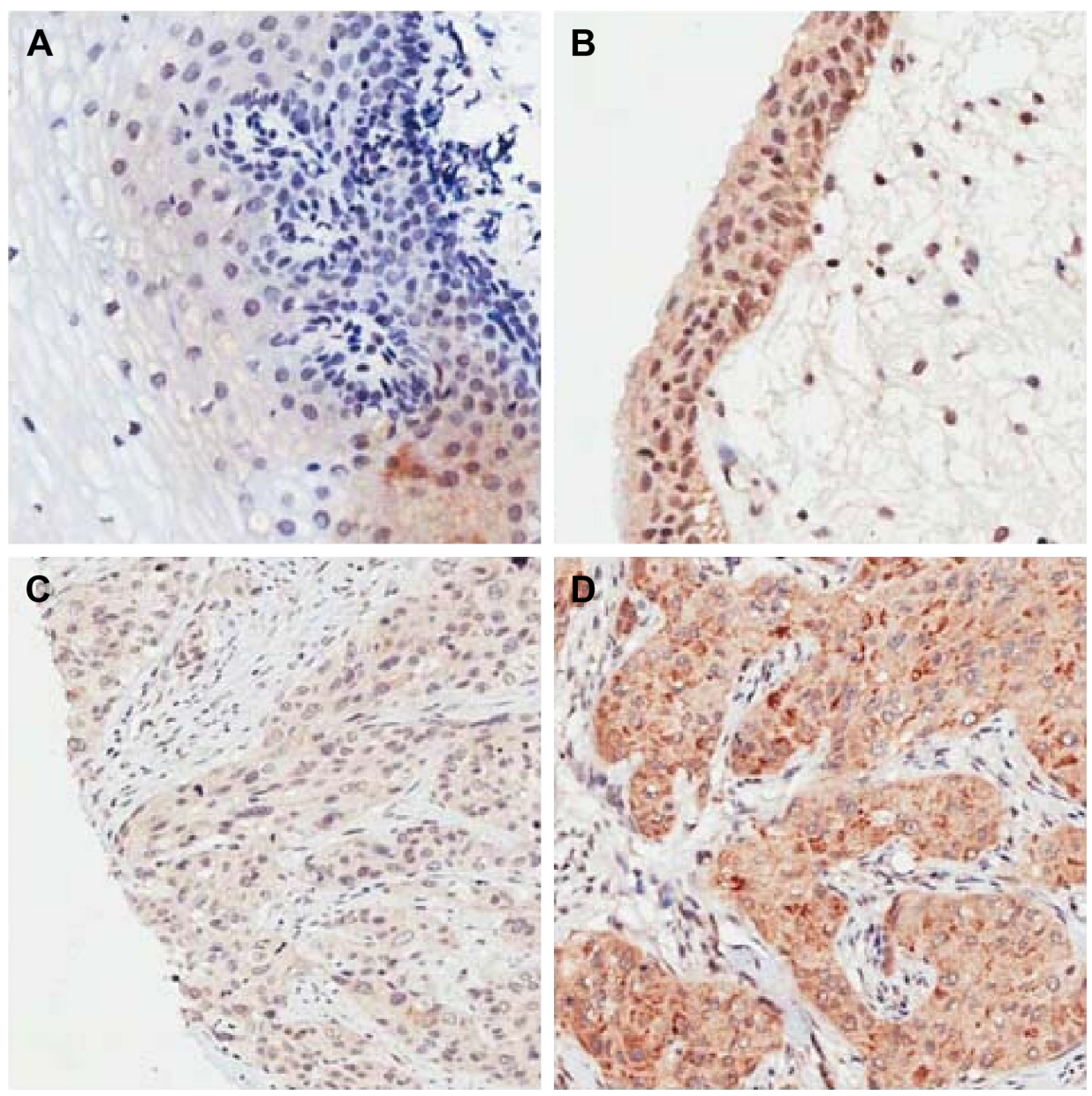

Figure 2 Expression of GRP78 was detected by immunohistochemistry.

Notes: (A) Negative staining of GRP78 in normal esophageal epithelium (400x). (B) High expression of GRP78 in normal esophageal epithelium (400x). (C) Low expression of GRP78 in ESCC tissues (400x). (D) High expression of GRP78 in ESCC tissues (400x).

Abbreviations: ESCC, esophageal squamous cell carcinoma; GRP78, glucose-regulated protein 78 .

hazard model demonstrated that gender $(P=0.040)$, smoking status $(P=0.017)$, tumor size $(P=0.045)$, tumor invasion depth $(P=0.000)$, LN metastasis $(P=0.002)$, and GRP78 expression $(P=0.037)$ were significantly correlated with OS. These parameters were subjected to multivariate analysis, and the results suggested that tumor invasion depth $(P=0.002), \mathrm{LN}$ metastasis $(P=0.032)$, and GRP78 expression $(P=0.011)$ were independent prognostic factors of the OS of ESCC patients.

\section{GRP78 promotes the invasion and migration of ESCC cells}

TE-1 and CaEs-17 were transiently transfected with si-GRP78 or si-con for $48 \mathrm{~h}$ to explore the role of GRP78 in ESCC invasion and migration. The efficiency of transfection was confirmed through Western blot assay. GRP78 expression levels in both cell lines were more effectively reduced by si-GRP78 than by si-con $(P<0.05$; Figure 4$)$. Transwell migration and invasion assays were then performed to investigate the role of GRP78 in ESCC cell invasion and migration in vitro. The results revealed that the migratory activity of the si-GRP78-transfected TE-1 and CaEs-17 cells was remarkably reduced, GRP78 expression was suppressed in both cell lines, and si-GRP78 decreased their invasion into the Matrigel substrate.

\section{Discussion}

Metastasis, a result of cancer migration and invasion from primary site to distant site, is the major cause of cancerrelated deaths. ${ }^{18}$ Invasion and metastasis in many different human cancer types are correlated with GRP78 expression levels. ${ }^{19-21}$ In breast cancer, the GRP78 expression level can be considered as a biomarker to predict invasion and metastasis. ${ }^{22}$ In gastric cancer, GRP78 overexpression is 
Table I Correlation between the expression of GRP78 protein and clinicopathological features

\begin{tabular}{|c|c|c|c|c|c|}
\hline \multirow{2}{*}{$\begin{array}{l}\text { Clinicopathological } \\
\text { features }\end{array}$} & \multirow[t]{2}{*}{ Case } & \multicolumn{2}{|c|}{ GRP78 expression } & \multirow[t]{2}{*}{$\chi^{2}$} & \multirow[t]{2}{*}{$P$-value } \\
\hline & & Low (\%) & High (\%) & & \\
\hline $\begin{array}{l}\text { Total number of } \\
\text { patients }\end{array}$ & 92 & $26(28.3)$ & 66 (7I.7) & & \\
\hline Gender & & & & 0.237 & 0.627 \\
\hline Male & 67 & $18(26.9)$ & $49(73.1)$ & & \\
\hline Female & 25 & $8(32.0)$ & $17(68.0)$ & & \\
\hline Age (years) & & & & 1.930 & 0.165 \\
\hline$<65$ & 46 & $16(34.8)$ & $30(65.2)$ & & \\
\hline$\geq 65$ & 46 & $10(21.7)$ & $36(78.3)$ & & \\
\hline Smoking status & & & & 0.106 & 0.745 \\
\hline None & 33 & $10(30.3)$ & $23(69.7)$ & & \\
\hline Yes & 59 & $16(27.1)$ & 43 (72.9) & & \\
\hline Alcohol consumption & & & & 0.531 & 0.466 \\
\hline status & & & & & \\
\hline None & 37 & $12(32.4)$ & $25(67.6)$ & & \\
\hline Yes & 55 & $14(25.5)$ & $4 I(74.5)$ & & \\
\hline Tumor location & & & & 1.582 & 0.453 \\
\hline Upper & 2 & I (50) & I (50) & & \\
\hline Middle & 73 & $22(30.1)$ & $51(69.9)$ & & \\
\hline Lower & 17 & $3(17.6)$ & $14(82.4)$ & & \\
\hline Histological grade & & & & 3.314 & 0.191 \\
\hline GI & 10 & I (10) & $9(90)$ & & \\
\hline $\mathrm{G} 2$ & 65 & $18(27.7)$ & $47(72.3)$ & & \\
\hline G3 & 17 & $7(4 \mid .2)$ & $10(58.8)$ & & \\
\hline Tumor size $(\mathrm{cm})$ & & & & 1.930 & 0.165 \\
\hline$<4$ & 46 & $16(34.8)$ & $30(65.2)$ & & \\
\hline$\geq 4$ & 46 & $10(21.7)$ & $36(78.3)$ & & \\
\hline Tumor invasion & & & & 1.551 & 0.213 \\
\hline TI-T2 & 30 & II (36.7) & $19(63.3)$ & & \\
\hline T3-T4 & 62 & $15(24.2)$ & 47 (75.8) & & \\
\hline Lymph node metastasis & & & & 4.429 & 0.035 \\
\hline No & 55 & $20(36.4)$ & $35(63.6)$ & & \\
\hline $\mathrm{N}+$ & 37 & $6(16.2)$ & $31(83.8)$ & & \\
\hline TNM stage & & & & 8.106 & 0.017 \\
\hline 1 & 10 & $6(60.0)$ & $4(40.0)$ & & \\
\hline II & 36 & $12(33.3)$ & $24(66.7)$ & & \\
\hline III & 46 & $8(17.4)$ & $38(82.6)$ & & \\
\hline
\end{tabular}

Note: Bold values indicate statistical significance $(P<0.05)$.

Abbreviation: GRP78, glucose-regulated protein 78 .

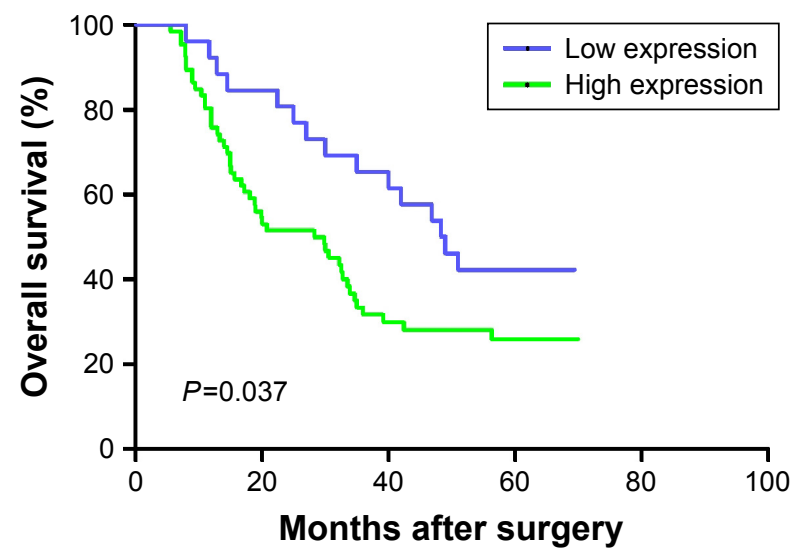

Figure 3 Association between the GRP78 expression levels and OS of ESCC patients. Note: The 5-year survival rates of esophageal cancer patients with low GRP78 expression levels were significantly higher than those with high GRP78 expression levels $(P=0.037)$.

Abbreviations: ESCC, esophageal squamous cell carcinoma; GRP78, glucoseregulated protein 78; OS, overall survival. correlated with LN metastasis. ${ }^{12}$ GRP78 overexpression in esophageal cancer is related to aggressive biological behavior, poor prognosis, and chemotherapy response. ${ }^{7,10}$ These findings indicated that targeting GRP78 may be a new therapeutic approach in esophageal cancer, but previous data were mainly derived from esophageal adenocarcinoma cases. ${ }^{9,10}$ Although the role of GRP78 in tumor progression has been examined in many human cancers, the invasion and metastasis of ESCC have yet to be fully elucidated. Therefore, we performed this study to analyze the expression and clinical implications of GRP78 in ESCC and explore the correlation of GRP78 expression with invasion and metastasis in ESCC.

In this study, the expression of GRP78 protein in primary ESCC tissues was examined through immunohistochemistry and Western blot. The correlation of GRP78 expression with clinicopathological variabilities and prognosis was also analyzed. Our results indicated that the GRP78 expression significantly differed between ESCC and adjacent normal tissue samples. Immunohistochemical analysis revealed that the GRP78 expression in the cytoplasm of the ESCC cells was higher than that in the cytoplasm of the corresponding normal esophageal tissues. These findings are consistent with those described in previous studies ${ }^{7,13,23}$ and of potential clinical importance for early diagnosis. Further analysis showed that high GRP78 expression was associated with LN metastasis and advanced clinical stage. These results also agree with those observed by Langer et al. ${ }^{9}$ Zhao et al ${ }^{24}$ performed a log-rank test and found that patients with high GRP78 expression exhibit poor prognosis. To establish the prognostic role of GRP78, we precluded ESCC patients with stage IV and carried out univariate and multivariate analyses. Our finding demonstrated that the patients with low GRP78 expression in tumors tissue achieved a higher OS than those of patients with high tumor expression $(P=0.037)$. The multivariate Cox analysis of GRP78 expression and other parameters showed that GRP78 expression was an independent indicator of survival in this research. This result suggested that GRP78 might play an important role in disease aggressiveness and serve as a novel prognostic marker for patients with ESCC. As such, we further explored the biological role of GRP78 in ESCC development in vitro.

We further performed GRP78 siRNA knockdown in two types of GRP78-overexpressing ESCC cell lines to analyze the effect of GRP78 expression on their invasion and metastatic capacities. Our results showed that the invasion and migration capacities of these two cancer cell lines 
Table 2 Univariate and multivariate analyses of different prognostic factors in patients with ESCC by Cox regression model

\begin{tabular}{|c|c|c|c|c|}
\hline \multirow{2}{*}{$\begin{array}{l}\text { Clinicopathological } \\
\text { features }\end{array}$} & \multicolumn{2}{|c|}{ Univariate analysis } & \multicolumn{2}{|c|}{ Multivariate analysis } \\
\hline & $P$-value & HR (95\% Cl) & $P$-value & HR (95\% Cl) \\
\hline \multicolumn{5}{|l|}{ Gender } \\
\hline Male vs female & 0.040 & $0.522(0.283-0.965)$ & $0.24 I$ & $1.447(0.780-2.684)$ \\
\hline \multicolumn{5}{|l|}{ Age (years) } \\
\hline$<65$ vs $\geq 65$ & 0.265 & $1.329(0.806-2.189)$ & & \\
\hline \multicolumn{5}{|l|}{ Smoking status } \\
\hline None vs yes & 0.017 & $0.514(0.293-0.899)$ & 0.147 & $0.652(0.366-1.162)$ \\
\hline \multicolumn{5}{|l|}{ Alcohol consumption status } \\
\hline None vs yes & 0.135 & $0.674(0.40 \mathrm{I}-\mathrm{I} .136)$ & & \\
\hline \multicolumn{5}{|l|}{ Tumor location } \\
\hline Upper vs middle vs lower & 0.241 & $0.655(0.323-1.329)$ & & \\
\hline \multicolumn{5}{|l|}{ Histological grade } \\
\hline GI vs $G 2$ vs G3 & 0.173 & $0.733(0.468-1.146)$ & & \\
\hline \multicolumn{5}{|l|}{ Tumor size $(\mathrm{cm})$} \\
\hline$<4$ vs $\geq 4$ & 0.045 & 1.685 (1.013-2.805) & 0.131 & I.503 (0.886-2.550) \\
\hline \multicolumn{5}{|l|}{ Tumor invasion } \\
\hline TI-T2 vs T3-T4 & 0.000 & $3.315(1.756-6.256)$ & 0.002 & $2.869(1.489-5.526)$ \\
\hline \multicolumn{5}{|l|}{ Lymph node metastasis } \\
\hline $\mathrm{N} 0$ vs $\mathrm{N}+$ & 0.002 & $2.267(\mathrm{I} .367-3.76 \mathrm{I})$ & 0.032 & $1.689(1.004-2.84 I)$ \\
\hline \multicolumn{5}{|l|}{ GRP78 expression } \\
\hline Low vs high & 0.037 & $1.949(1.069-3.551)$ & 0.011 & $0.475(0.25 \mathrm{I}-0.898)$ \\
\hline \multicolumn{5}{|l|}{ Adjuvant therapy } \\
\hline None vs yes & 0.173 & $1.415(0.859-2.322)$ & & \\
\hline
\end{tabular}

Note: Bold values indicate statistical significance $(P<0.05)$.

Abbreviations: ESCC, esophageal squamous cell carcinoma; GRP78, glucose-regulated protein 78; HR, hazard ratio; Cl, confidence interval.

were lower in the GRP78 siRNA knockdown group than in the control group. This observation is consistent with that presented by Zhao et al. ${ }^{24}$

Although overexpression of GRP78 has been reported to be associated with invasion and metastasis in many human cancers, the exact molecular mechanism remains unclear. Dong et a ${ }^{25}$ explored the role of GRP78 in a tumor microenvironment and found that tumor growth and angiogenesis are suppressed in GRP78 ${ }^{+-}$mice in the early phase but not in the late phase. Therefore, GRP78 overexpression occurring in early-stage ESCC may be a reaction to an early response induced by hosts' immune systems. By contrast, stress factors against tumors may contribute to the upregulation of GRP78 expression in the metastatic stage. ${ }^{9}$ Cell migration and adhesive signaling are regulated by an essential nonreceptor tyrosine kinase named FAK. ${ }^{20}$ The activation and activity of FAK may be an underlying mechanism by which GRP78 promotes the invasion of cells in hepatocellular carcinoma ${ }^{13}$ and pancreatic cancer. ${ }^{26} \beta 1$-Integrin is an important molecule involved in cell matrix adhesion and FAK activity. ${ }^{27}$ GRP78regulated FAK activity may be mediated by $\beta 1$-integrin, and GRP78 may facilitate colorectal cancer cell invasion by interacting with the urokinase-type plasminogen activator (uPA)-uPA receptor (uPAR) system. ${ }^{20}$ Vimentin is a key molecule involved in epithelial-to-mesenchymal transition.
However, GRP78 may play a suppressor role in modulating cell migration by enhancing vimentin expression levels in hepatocellular cancer. ${ }^{19}$ Therefore, the role of GRP78 in cell invasion and migration might vary across different cancer types.

However, our study is characterized by several potential limitations. For instance, this study applied a retrospective and single-hospital design. Another limitation is the relatively small sample size. Future large-scale, multicenter, and prospective studies should be performed to identify the prognostic value of GRP78 in ESCC.

\section{Conclusion}

We observed that GRP78 expression was higher in ESCC tissues than in normal esophageal mucosa. High GRP78 expression was associated with LN metastasis and advanced clinical stage. It could also act as an independent indicator of poor OS in ESCC. High GRP78 expression also enhanced the invasion and migration abilities of ESCC cells. GRP78 might be a potential anti-invasion and migration target in ESCC treatment. Further studies on the mechanism of the invasion and metastasis of GRP78 should be conducted to clarify the role of GRP78 in ESCC, improve the prognostic status of affected patients, and generate individualized cancer treatments. 
A

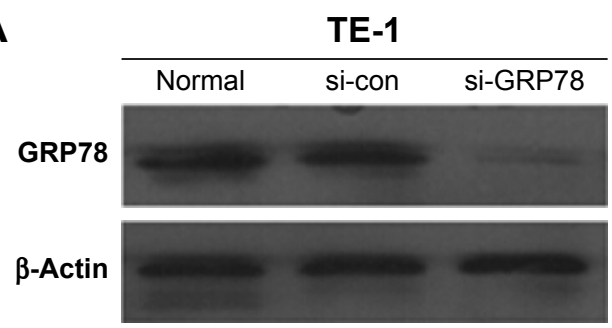

TE-1
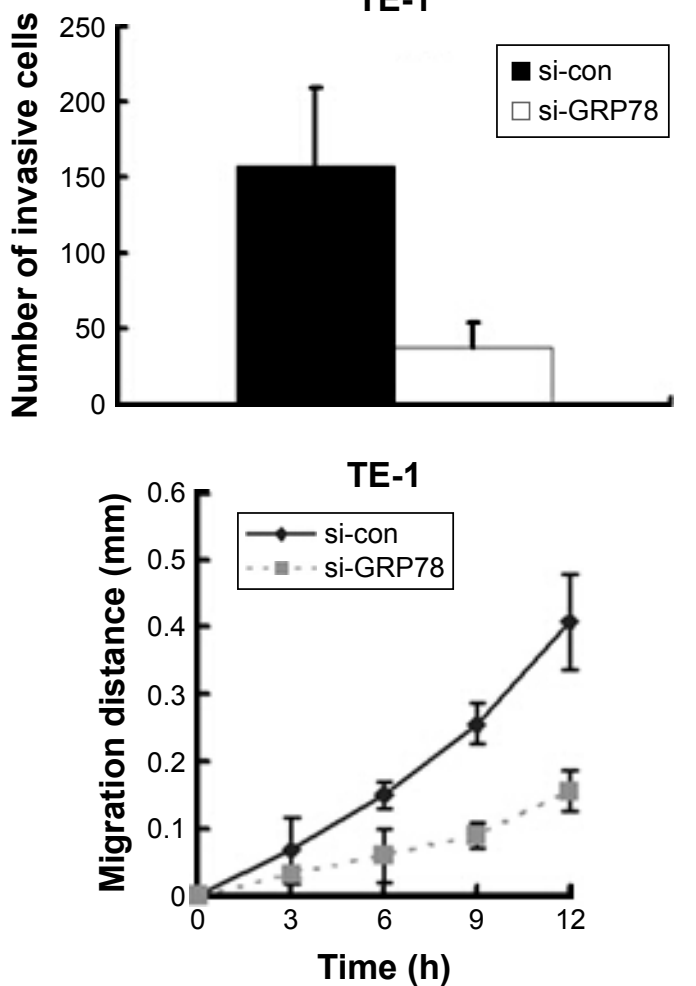

B

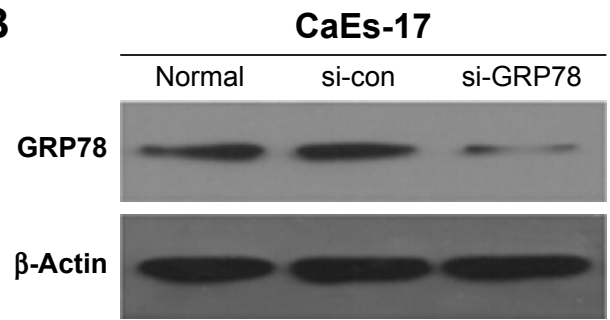

CaEs-17
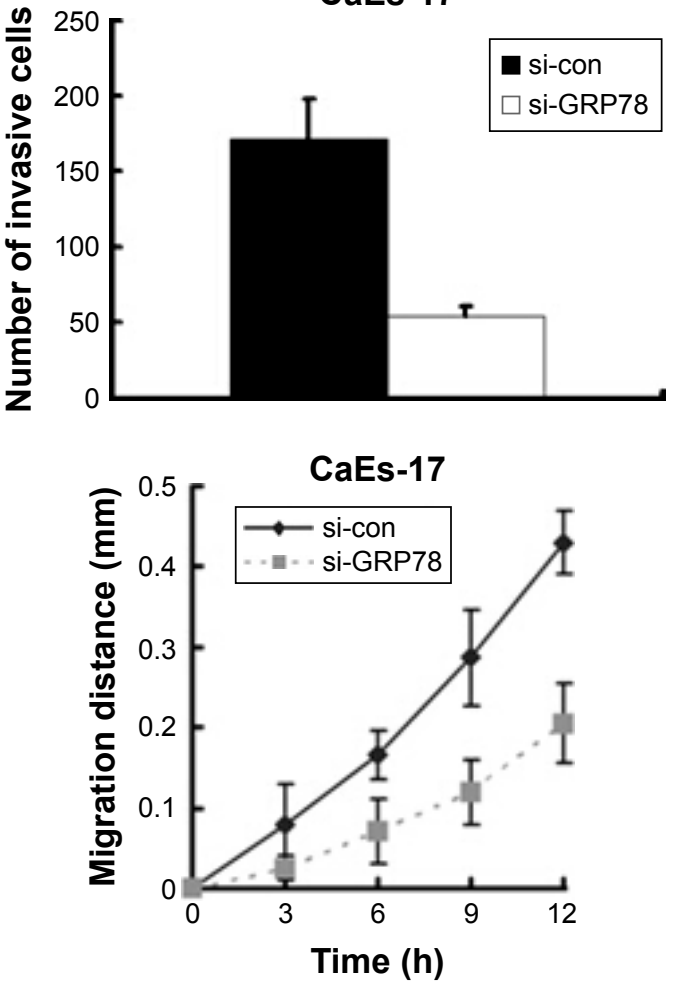

Figure 4 Downregulating GRP78 expression could inhibit the invasion and migration abilities of ESCC cells in vitro.

Notes: Matrigel invasion assay and Transwell migration assay of the ESCC cell lines. TE-I and CaEs-17 transfected with GRP78 siRNA (si-GRP78) and negative control siRNA (si-con). (A) TE-I cells and (B) CaEs-I7 cells.

Abbreviations: ESCC, esophageal squamous cell carcinoma; GRP78, glucose-regulated protein 78; siRNA, small interfering RNA.

\section{Acknowledgments}

This study was supported by the National Key Clinical Specialist Construction Programs of China (no 2013-54) and the National Natural Science Foundation of China (81071981).

\section{Disclosure}

The authors report no conflicts of interest in this work.

\section{References}

1. Kamangar F, Dores GM, Anderson WF. Patterns of cancer incidence, mortality, and prevalence across five continents: defining priorities to reduce cancer disparities in different geographic regions of the world. $J$ Clin Oncol. 2006;24(14):2137-2150.

2. Pennathur A, Gibson MK, Jobe BA, Luketich JD. Oesophageal carcinoma. Lancet. 2013;381(9864):400-412.

3. Little E, Ramakrishnan M, Roy B, Gazit G, Lee AS. The glucoseregulated proteins (GRP78 and GRP94): functions, gene regulation, and applications. Crit Rev Eukaryot Gene Expr. 1994;4(1):1-18.
4. Lee AS. The glucose-regulated proteins: stress induction and clinical applications. Trends Biochem Sci. 2001;26(8):504-510.

5. Pyrko P, Schonthal AH, Hofman FM, Chen TC, Lee AS. The unfolded protein response regulator GRP78/BiP as a novel target for increasing chemosensitivity in malignant gliomas. Cancer Res. 2007;67(20):9809-9816.

6. Xing X, Lai M, Wang Y, Xu E, Huang Q. Overexpression of glucoseregulated protein 78 in colon cancer. Clin Chim Acta. 2006;364(1-2): 308-315.

7. Du XL, Hu H, Lin DC, et al. Proteomic profiling of proteins dysregulted in Chinese esophageal squamous cell carcinoma. $J$ Mol Med (Berl). 2007;85(8):863-875.

8. Zhang XX, Li HD, Zhao S, et al. The cell surface GRP78 facilitates the invasion of hepatocellular carcinoma cells. Biomed Res Int. 2013;2013: 917296.

9. Langer R, Feith M, Siewert JR, Wester HJ, Hoefler H. Expression and clinical significance of glucose regulated proteins GRP78 (BiP) and GRP94 (GP96) in human adenocarcinomas of the esophagus. BMC Cancer. 2008;8:70.

10. Slotta-Huspenina J, Wolff C, Drecoll E, et al. A specific expression profile of heat-shock proteins and glucose-regulated proteins is associated with response to neoadjuvant chemotherapy in oesophageal adenocarcinomas. Br J Cancer. 2013;109(2):370-378. 
11. Slotta-Huspenina J, Berg D, Bauer K, et al. Evidence of prognostic relevant expression profiles of heat-shock proteins and glucoseregulated proteins in oesophageal adenocarcinomas. PLoS One. 2012; 7(7):e41420.

12. Zhang J, Jiang Y, Jia Z, et al. Association of elevated GRP78 expression with increased lymph node metastasis and poor prognosis in patients with gastric cancer. Clin Exp Metastasis. 2006;23(7-8):401-410.

13. Su R, Li Z, Li H, et al. Grp78 promotes the invasion of hepatocellular carcinoma. BMC Cancer. 2010;10:20.

14. Flejou JF. Classification OMS 2010 des tumeurs digestives : la quatrième édition. [WHO classification of digestive tumors: the fourth edition]. Ann Pathol. 2011;31(5 suppl):S27-S31. French.

15. Rice TW, Blackstone EH, Rusch VW. 7th edition of the AJCC cancer staging manual: esophagus and esophagogastric junction. Ann Surg Oncol. 2010;17(7):1721-1724.

16. Zu K, Bihani T, Lin A, Park YM, Mori K, Ip C. Enhanced selenium effect on growth arrest by BiP/GRP78 knockdown in p53-null human prostate cancer cells. Oncogene. 2006;25(4):546-554.

17. Sun GG, Wang YD, Cheng YJ, Hu WN. BTG1 underexpression is an independent prognostic marker in esophageal squamous cell carcinoma. Tumour Biol. 2014;35(10):9707-9716.

18. Fidler IJ. Critical determinants of metastasis. Semin Cancer Biol. 2002; 12(2):89-96.

19. Wei PL, Kuo LJ, Wang W, et al. Silencing of glucose-regulated protein 78 (GRP78) enhances cell migration through the upregulation of vimentin in hepatocellular carcinoma cells. Ann Surg Oncol. 2012;19(suppl 3): S572-S579.
20. Li Z, Zhang L, Zhao Y, et al. Cell-surface GRP78 facilitates colorectal cancer cell migration and invasion. Int J Biochem Cell Biol. 2013;45(5):987-994.

21. Zheng HC, Takahashi H, Li XH, et al. Overexpression of GRP78 and GRP94 are markers for aggressive behavior and poor prognosis in gastric carcinomas. Hum Pathol. 2008;39(7):1042-1049.

22. Dong D, Ni M, Li J, et al. Critical role of the stress chaperone GRP78/ $\mathrm{BiP}$ in tumor proliferation, survival, and tumor angiogenesis in transgene-induced mammary tumor development. Cancer Res. 2008; 68(2):498-505.

23. Yang L, Su M, Ma Y, Hao X. Novel immunohistochemical monoclonal antibody against human glucose-regulated protein 78. Hybridoma (Larchmt). 2011;30(6):559-562.

24. Zhao G, Kang J, Jiao K, et al. High expression of GRP78 promotes invasion and metastases in patients with esophageal squamous cell carcinoma. Dig Dis Sci. 2015;60(9):2690-2699.

25. Dong D, Stapleton C, Luo B, et al. A critical role for GRP78/BiP in the tumor microenvironment for neovascularization during tumor growth and metastasis. Cancer Res. 2011;71(8):2848-2857.

26. Yuan XP, Dong M, Li X, Zhou JP. GRP78 promotes the invasion of pancreatic cancer cells by FAK and JNK. Mol Cell Biochem. 2015; 398(1-2):55-62.

27. Lawson C, Lim ST, Uryu S, Chen XL, Calderwood DA, Schlaepfer DD FAK promotes recruitment of talin to nascent adhesions to control cell motility. J Cell Biol. 2012;196(2):223-232.
OncoTargets and Therapy

\section{Publish your work in this journal}

OncoTargets and Therapy is an international, peer-reviewed, open access journal focusing on the pathological basis of all cancers, potential targets for therapy and treatment protocols employed to improve the management of cancer patients. The journal also focuses on the impact of management programs and new therapeutic agents and protocols on

\section{Dovepress}

patient perspectives such as quality of life, adherence and satisfaction. The manuscript management system is completely online and includes a very quick and fair peer-review system, which is all easy to use. Visit http://www.dovepress.com/testimonials.php to read real quotes from published authors. 